pathological lesion was described in seven cases; five showed neurofibromatous infiltration or compression. In Miura's case, ${ }^{9}$ neurofibromatous infiltration of the subclavian wall was not found, but there was evidence of vascular dysplasia, consisting of decreased elastic fibres and deranged smooth muscle. In the absence of a demonstrable intrathoracic tumour, we believe that a similar vascular dysplasia was responsible for haemorrhage in our case.

Out of 18 patients reported in the literature, ${ }^{4-9}$ plus our own case, the four patients who had no surgical intervention died. Of the remaining 15 who underwent thoracotomy, nine survived.

Von Recklinghausen's disease is a relatively common disorder and this syndrome of spontaneous haemothorax deserves wider rec-

$1 \mathrm{Lie}$ JT. Vasculitis look-alikes and pseudovasculitis syndromes. Curr Diagn Pathol 1995;2:78-85.

2 Greene JF, Fitzwater JE, Burgess J. Arterial lesions associated with neurofibromatosis. Am $\mathrm{f}$ Clin Pathol 1974;62:481-7.

3 Reubi F. Neurofibromatose et lésions vasculaires (Neurofibromatosis and vascular lesions). Schweiz Med Wochenschr 1945;75:463-5.

4 Leier CV, DeWan CJ, Anatasia LF. Fatal hemorrhage as a complication of neurofibromatosis. Vasc Surg 1972;6:98101 .

5 Butchart EG, Grotte GJ, Barnsley WC. Spontaneous rupture of an intercostal artery in a patient with neurofibromatosis and scoliosis. $\mathcal{F}$ Thorac Cardiovasc Surg 1975;69: 919-21. ognition in view of its high mortality. To the pathologist, it is another addition to the ever-lengthening list of causes of sudden death, but knowledge of the syndrome will help to explain an otherwise puzzling accumulation of blood behind the pleura or within the mediastinum and guide the search for the cause. For clinicians, recognition of the syndrome should lead to urgent referral to the nearest cardiothoracic centre. The configuration of the radiological opacity and the ease with which frank blood is withdrawn on paracentesis should suggest the diagnosis in a patient with cutaneous manifestations of neurofibromatosis.

We are grateful to Mrs Gaynor Francis for secretarial assistance, and to Dr Kim Harrison, Chest Physician at Morriston Hospital, for drawing our attention to the report by Miura et al. ${ }^{9}$

6 Larrieu AJ, Hashimoto SA, Allen P. Spontaneous massive haemothorax in von Recklinghausen's disease. Thorax 1982; 37:151-2.

7 Brady DB, Bolan JC. Neurofibromatosis and spontaneous hemothorax in pregnancy. Two case reports. Obstet Gynecol 1984;63(suppl 3):35-38S.

8 Fuyuno G, Kobayashi R, Iga R, et al. A case of von Recklinghausen's disease associated with a hemothorax due to a rapidly growing malignant Schwannoma. fap $\mathcal{f}$ Thorac Dis 1995;33:682-5

9 Miura H, Taira 0, Uchida 0, Usuda J, Hirai S, Kato H. Spontaneous haemothorax associated with von Recklinghausen's disease: review of occurrence in Japan. Thorax 1997;52:577-8.

\title{
The best management for 'crescendo biliary colic' is urgent laparoscopic cholecystectomy
}

\author{
G S M Robertson, S A Wemyss-Holden, G J Maddern
}

\section{Summary}

Gallbladder disease due to stones is well recognised as falling into two categories, presenting with either chronic symptoms or developing acute cholecystitis or other complications. We describe an intermediate group of 14 patients (11 women, three men, median age 31 years) presenting with 4-14 days of at least daily attacks of resolving biliary colic, who underwent early laparoscopic cholecystectomy within 24 hours of presentation. None had any evidence of acute inflammation, either at laparoscopy or on histology. Their surgery was straightforward with operating times ranging from 35-80 minutes and no complications. Patients with 'crescendo biliary colic' are often young women who can rarely afford invalidity. Rather than the current practice of analgesia for each attack and elective surgery weeks later, they are optimally managed by urgent laparoscopic cholecystectomy, preventing the development of complications and minimising the need for further medical involvement.

Keywords: gallstones; biliary colic; laparoscopic cholecystectomy

Patients with gallstones are usually regarded as having either intermittent biliary colic resulting in 'chronic' inflammatory changes or 'acute' complications such as cholecystitis. ${ }^{1}$ Most surgeons and general practitioners will, however, be familiar with a group of patients developing acute cholecystitis or other complications of gallstones, who give a history of dramatically worsening biliary colic over the preceding couple of weeks. Such patients have frequently seen the emergency services with episodes of pain but in the UK are rarely admitted until their pain fails to resolve and acute cholecystitis or other complications supervene. Even when admitted, they are usually pain-free the following day and are discharged pending elective surgery or further emergency admission. 
Over the last 9 months we have prospectively audited a policy of early laparoscopic cholecystectomy in all patients admitted to our Unit as an emergency with gallstone-related symptoms. The majority have been patients with acute cholecystitis (17), empyema of the gallbladder (eight), or pancreatitis (three), or have been elective admissions (88). There has also been a group of 14 patients $(10 \%)$ with what we have christened 'crescendo biliary colic', admitted with up to 2 weeks of daily attacks of uncomplicated biliary colic, often on a background of previous less frequent symptoms. This report reviews their management and its implications based on our experience.

\section{Methods and results}

Eleven women and three men, median age 31 (range 23-81) years, were admitted with a median history of 7 (range 4-14) days of at least daily attacks of biliary colic. All of them had proven gallbladder stones of varying sizes on ultrasound with only two showing any evidence of gallbladder wall thickening. Three patients had slightly elevated liver function tests, two of whom had a dilated common bile duct ( 8 and $9.6 \mathrm{~mm}$ ) on ultrasound. On examination, each was apyrexial, with a normal white cell count, range 4.7-9.2 $\times 10^{9} / 1$ (normal 4.0-10.0 $\times 10^{9} / 1$ ) and normal amylase levels.

At laparoscopic cholecystectomy within 24 hours of admission, there was evidence of oedema in the gallbladder wall in four patients but no other evidence of acute inflammation. Laparoscopic cholecystectomy was straightforward taking a median of 56 (range 35-80) minutes, with no gallbladder perforations or conversions. The three patients with elevated liver function tests underwent normal peroperative cholangiography. Seven patients were discharged the following day, the rest stayed a further day, there were no complications. Histology of the gallbladder showed gallstonerelated changes in each case with no evidence of acute inflammation, wall thickness ranged from 3-7 mm (median $3 \mathrm{~mm}$ ) microscopically.

1 Hermann RE. Surgery for acute and chronic cholecystitis Surg Clin North Am 1990;70:1263-75.

2 Fried GM, Barkun JS, Sigman HH, et al. Factors determin ing conversion to laparotomy in patients undergoing laparoscopic cholecystectomy. Am ₹ Surg 1994;167:35-41.

\section{Learning points}

- patients with gallstones can suffer escalating or crescendo attacks of biliary colic

- such patients are often young women with families to care for

- laparoscopic cholecystectomy at this stage is straightforward with a short hospital stay

- if managed conservatively such patients are likely to develop complications of their gallstones, occupy more health service resources before inevitable surgery, and have continuing problems coping with family commitments

- the Health Service in the UK needs to adapt to refer such patients for urgent laparoscopic cholecystectomy

\section{Discussion}

Patients with frequent (crescendo) biliary colic due to a gallstone recurrently obstructing the cystic duct or Hartmann's pouch are inevitably at high risk of developing acute cholecystitis or more serious complications as the stone is passed into the common bile duct. Laparoscopic cholecystectomy then becomes more difficult requiring more surgical experience ${ }^{2}$ and operating time, ${ }^{3}$ and carrying a higher rate of conversion to open surgery ${ }^{2}{ }^{3}$ and a greater risk of complications. ${ }^{3}$

Our experience suggests that such patients, rather than being managed at home with analgesia, should be admitted for early straightforward laparoscopic cholecystectomy the following day. Such a policy is highly acceptable to patients who, in our experience, are often women with young families, and should minimise further health service involvement in a group of patients who inevitably require surgery sooner or later. Logistically this policy requires the ability to ultrasound the gallbladder, ideally on the day of admission, and the flexibility of theatre services, including surgeons, to accommodate their surgery the following day. It therefore requires a change in both referral and surgical practice.

3 Kum C-K, Eypasch E, Lefering R, Paul A, Neugebauer E, Troidl H. Laparoscopic cholecystectomy for acute cholecystitis: is it really safe? World $\mathcal{F}$ Surg 1996;20:43-9. 